\title{
A Boundary Lubrication Model And Experimental Study Considering ZDDP Tribofilms On Reciprocating Friction Pairs
}

\section{Bugao Lyu}

Shanghai Jiaotong University

Limin Zhang

China North Engine Research Institute

Xianghui Meng ( $\nabla$ xhmeng@sjtu.edu.cn )

Shanghai Jiaotong University https://orcid.org/0000-0003-2387-1186

Chengen Wang

Shanghai Jiaotong University

\section{Research Article}

Keywords: Boundary lubrication, Tribofilm, ZDDP, friction, Wear

Posted Date: February 7th, 2022

DOI: https://doi.org/10.21203/rs.3.rs-1320794/v1

License: (c) (i) This work is licensed under a Creative Commons Attribution 4.0 International License. Read Full License 


\title{
A boundary lubrication model and experimental study considering ZDDP tribofilms on reciprocating friction pairs
}

\author{
Bugao Lyu ${ }^{1}$, Limin Zhang ${ }^{2}$, Xianghui Meng ${ }^{1 *}$, Chengen Wang ${ }^{1}$ \\ 1. School of Mechanical Engineering, Shanghai Jiaotong University, Shanghai 200240, People's \\ Republic of China.
}

2. China North Engine Research Institute, Tianjin 300400, People's Republic of China.

\begin{abstract}
Boundary lubrication state may dominate the friction pairs operating under severe conditions, yet its mechanism is not clearly understood and related numerical models are still lacking. A boundary lubrication model considering zinc dialkyldithiophosphate (ZDDP) tribofilms, which impact the friction and wear performances, is developed in this study. A series of reciprocating experiments are conducted to verify this model and also to investigate the effects of the tribofilm on friction and wear under various temperatures and loads. Moreover the experimental data are employed to modify the tribofilm remove model, which enables the present boundary lubrication model to be applied under a wide range of loads. The results show that the friction force and wear depth both decline with the increasing lubricant temperature due to a thicker tribofilm formed. As the load becomes heavier, the wear depth keeps increasing, while the tribofilm thickness first increases then decreases.
\end{abstract}

Key words: Boundary lubrication; Tribofilm; ZDDP; friction; Wear

\section{Introduction}

Nowadays friction pairs are designed to be more and more compact, whilst their operating conditions are becoming more and more severe for a higher power efficiency. As a result, the friction pairs are inevitable to experience or even maintain boundary lubrication state under the harsh

${ }^{*}$ Corresponding author: Xianghui Meng, School of Mechanical Engineering, Shanghai Jiaotong University, No. 800, Dongchuan Road, Shanghai 200240, People's Republic of China, Email: xhmeng@sjtu.edu.cn 
operating conditions [1]. In this situation, the contacting surfaces are not well lubricated by a fluid film [2] but separated by a protective surface-bonded tribofilm in the presence of lubricant additives. The tribofilm will impact the fluid lubrication, asperity contact, friction, and wear during surface rubbing [3]. However a numerical model to describe these effects is still lacking, especially for engineering friction pairs under dynamic working conditions. Therefore a boundary lubrication model considering the tribofilms, which will be calibrated and verified by experiments, is developed in this study.

One of the most efficient tribofilm to reduce surface wear is the ZDDP tribofilm and many studies have been conducted on its formation mechanism, friction and wear performances. The ZDDP tribofilm tightly bonds to the rubbing surfaces with a thickness as high as $200 \mathrm{~nm}$ [4]. It consists of rough, patchy, pad-like features that are composed of pyro- or orthophosphate glasses in the bulk, with an outer nanoscale layer of zinc polyphosphates and a sulfur-rich layer near the substrate surface [3]. It is demonstrated that a high contact pressure and lubricant temperature can promote the formation of ZDDP tribofilms [5], but it will be inhibited if the pressure is too high [6]. Gosvami et al. revealed that the tribofilm growth was stress-dependent by single-asperity sliding contact experiments and its growth rate fitted a stress-activated Arrhenius model [7]. They assumed this driving stress was normal pressure [7]. While Zhang and Spikes argued that it was primarily the shear stress [4], and they showed that tribofilms could grow even under full film lubrication where there was no asperity contact if only the shear stress was high enough [8]. Zhang et al. also studied the boundary friction of ZDDP tribofilms in both rolling/sliding and pure sliding contacts [9]. They found that the coefficient of friction $(\mathrm{CoF})$ of the tribofilm depended on the alkyl structure of the ZDDP. It is incontrovertible that ZDDP tribofilms can efficiently mitigate surface wear especially at extreme pressure, yet studies also found that oils with higher ZDDP concentrations produced more micro pitting [10]. The stress-activated model for the tribofilm growth is widely accepted. However, the process of tribofilm remove is still under debate and there is no reliable equation that can model it.

Most researches on the ZDDP tribofilm have been conducted experimentally, while a practical boundary lubrication model for engineering friction pairs has not yet been developed due to its complex and dynamic processes. Nevertheless, there are still several studies striving to model the tribofilm formation and its effects using numerical methods. Ghanbarzadeh et al. proposed a semideterministic wear model considering the effect of ZDDP tribofilms [11]. In their model, the contact pressure was calculated by the elastic-perfectly plastic theory and the wear was evaluated by a modified Archard's wear model [12] whose wear coefficient was varied with the tribofilm growth. Later Akchurin et al. also developed a model for the simulation of the tribofilm formation by integrating a boundary element method-based contact model and a stress-activated Arrhenius tribofilm growth equation [13]. The surface wear in the presence of tribofilms was regarded as mild wear resulting from the atom loss of the substrate material by tribofilm remove. Considering both fluid lubrication and the tribochemistry of tribofilms, Azam proposed a deterministic mixed lubrication model where the tribochemistry, lubrication, and asperity contact were coupled [14]. Similarly, a modified Archard's wear model was applied in their work and the asperity contact pressure was used to estimate surface wear. Recently, Chen et al. modeled the formation and remove of ZDDP tribofilm on rough surfaces, and applied this model for the cylinder liner surface of an internal combustion engine (ICE) [15]. They simulated the tribofilm growth on both the top dead center (TDC) and bottom dead center (BDC), which induced a distinguished wear topography at the 
end of the simulation. Yet these models/simulations are all based on deterministic asperity contacts, which is quite time-consuming when coupled with the hydrodynamic lubrication considering the simulation time up to several hours. Besides the friction force in the presence of tribofilms is not predicted in these simulations.

Therefore, a boundary lubrication model, in which the ZDDP tribofilm growth and remove are considered, will be presented in this study to capture its effects on both friction and wear performances. In this model, the asperity contact pressure is obtained using a statistical contact model, which is more practical for engineering friction pairs. Then experiments for reciprocating friction pairs under various conditions are conducted to calibrate and verify the presented model. Moreover a modified tribofilm remove model with an impact factor reflecting asperity contact state is proposed based on the experiments results. This study can help to understand the effects of tribofilm formation on friction and wear under boundary lubrication.

\section{Boundary lubrication model}

The research on boundary lubrication has commenced for a complete century ever since it was first systematically studied by Hardy [16] in 1922. It is believed that from a macroscopic perspective, the rubbing surfaces of friction pairs working under boundary lubrication are separated by thin films, which are called boundary films. The boundary films can be generally classified into absorption film and reaction film [17]. The adsorption film by physisorption or chemisorption is not considered in the present study because it is easily desorbed under loaded conditions [1]. As for the main topic in the present study, the ZDDP tribofilm growth on the rubbing surfaces by tribochemistry reactions between lubricant additives and substrate materials belongs to the latter. The ZDDP tribofilm partly covers the substrate surface with an evolving thickness and it will directly impact the contact state during rubbing. In the boundary lubrication model, the tribofilm as well as the substrate contact pressure are obtained by a layered statistical asperity contact model. The yielded results are then used to evaluate the boundary friction force and surface wear. At the same time, the tribofilm thickness is determined by the stress-activated growth model and the remove model.

\subsection{Layered statistical asperity contact model}

The tribofilm formed by anti-wear lubricant additives firmly bonds on the substrate surface. As the tribofilm growth, it will bear more and more applied load. Therefore the evolution of the tribofilm thickness will determine the asperity contact state. A layered contact model is proposed in this paper, where there are two layers, the tribofilm and substrate from the outside to the inside. The contact of two rough surfaces can be equivalent to one equivalent single rough surface in contact with a rigid flat surface, and it shows as: 


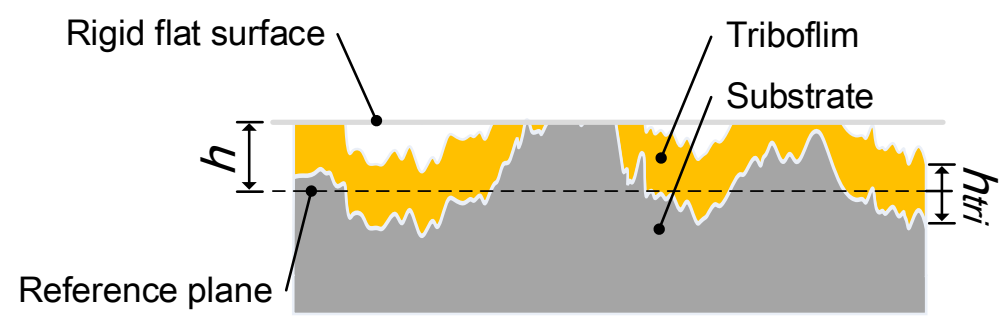

Fig. 1 The schematic diagram of the layered contact model.

The tribofilm thickness is represented by $h_{t r i}$ in Fig. 1 . The reference plane is located at the mean height of the substrate surface asperities. Considering the rough, patchy, and pad-like features of the ZDDP tribofilm, the substrate is partly and unevenly covered by the tribofilm. Thus the substrate-to-substrate contact and tribofilm-to-tribofilm contact exist simultaneously. Analogically, in the layered contact model the rigid surface will contact with the tribofilm and also penetrate it to contact with the substrate. Assume the separation between the rigid flat surface and the reference plane is $h$ and the probability density function of the asperity height is $\Phi(h)$, the substrate contact pressure $\left(p_{\text {sub }}\right)$ according to the statistical approach proposed by Greenwood and Tripp (GT) [18] is given as:

$$
p_{\text {sub }}(h)=\frac{16 \pi}{15}(\eta \beta \sigma)^{2} \sqrt{\frac{\sigma}{\beta}} E_{\text {sub }}^{\prime} \int_{h}^{\infty}(z-h)^{2.5} \phi(z) d z
$$

where $E_{\text {sub }}^{\prime}$ is the equivalent elastic modulus of the substrate material. $\sigma$ is the composite surface roughness, $\eta$ is the asperity density, and $\beta$ is the asperity radius. These parameters for a given friction pair are shown in section 3.1.

The tribofilm contact pressure $\left(p_{t r i}\right)$ is closely related to its thickness and is given as:

$$
p_{t r i}(h)=\frac{16 \pi}{15}(\eta \beta \sigma)^{2} \sqrt{\frac{\sigma}{\beta}} E_{t r i}^{\prime} \int_{h-h_{t r i}}^{h}(z-h)^{2.5} \phi(z) d z
$$

The integration from $h-h_{\text {tri }}$ to $h$ represents the contact proportion of the tribofilm and it is actually the difference by subtracting the contact proportion of the substrate (from $h$ to $\infty$ ) from the whole contact area (from $h-h_{t r i}$ to $\infty$ ). $E_{t r i}^{\prime}$ is the equivalent elastic modulus of the tribofilm, which ranges from 40 to $90 \mathrm{GPa}$ [19] and will be discussed in section 2.3.3.

The total asperity contact pressure is the sum of the substrate contact pressure and the tribofilm contact pressure:

$$
p_{a s p}=p_{s u b}+p_{t r i}
$$

The contact model above is simple yet logical and it depicts the process that the tribofilm will bear more applied load as it grows thicker. The model will be validated by comparing the simulation and experiment results in section 4.

The hydrodynamic pressure $\left(p_{l u b}\right)$ generated between rubbing surfaces is obtained by the average Reynolds equation [20]. The lubricant viscosity has a significant effect on the performance of the hydrodynamic pressure and varies with the pressure and temperature. It is characterized by Roelands's model [21]. Moreover the load is borne both by the fluid and asperities. The distributions of hydrodynamic pressure and asperity contact pressure are supposed to satisfy the load balance equation. It can be expressed as:

$$
w=\iint_{\mathrm{A}}\left(p_{\text {lub }}+p_{\text {asp }}\right) d A
$$

where $w$ is the applied load and $A$ is the contact area. 


\subsection{Boundary friction and wear evaluation}

Bowden and Tabor in the 1940s [22, 23] proposed a widely accepted model for boundary lubrication. In their model, the friction force of the rubbing surface comprises of the adhesive shear at the metallic contact areas and the viscous shear at the oil lubricating areas. This model in understanding the process of boundary lubrication is simple yet efficient. In the present model, the total friction force $F_{f r i}$ comprises of the tribofilm and substrate contact friction forces $F_{t r i}$ and $F_{\text {sub }}$, and the lubricant shear force $F_{l u b}$ :

$$
F_{f r i}=F_{t r i}+F_{\text {sub }}+F_{\text {lub }}
$$

The tribofilm and substrate contact friction forces $F_{t r i}, F_{s u b}$ are obtained as:

$$
\begin{aligned}
F_{t r i} & =\iint_{A} \mu_{t r i} p_{t r i} d A \\
F_{\text {sub }} & =\iint_{A} \mu_{\text {sub }} p_{\text {sub }} d A
\end{aligned}
$$

where $\mu_{t r i}$ and $\mu_{\text {sub }}$ are the CoFs of the tribofilm and substrate respectively. The CoF of the ZDDP tribofilm varies slightly within the range of $60-100{ }^{\circ} \mathrm{C}$ [24] and it is 0.1 used in [13]. In the present study, it is obtained by experimental calibration. The $\mathrm{CoF}$ of the substrate is obtained by dry sliding tribotest at a given friction pair.

For lubricant shear force $F_{l u b}$, it calculates as:

$$
F_{l u b}=\iint_{A}\left[\left(\phi_{f}+\phi_{f s}\right) \frac{\varepsilon u}{h}+\phi_{f p} \frac{h}{2} \frac{\partial p_{l u b}}{\partial x}\right] d A
$$

where the terms $\phi_{f}, \phi_{f s}$ and $\phi_{f p}$ are the friction-induced flow factors [20, 25]. $u$ is the sliding velocity, $\varepsilon$ is the lubricant viscosity, and $x$ is the coordinate parallel to the sliding direction.

In the boundary lubrication, the substrate is patchily covered by the tribofilm, and substrateto-substrate contact and tribofilm-to-tribofilm contact both exist. Therefore, the loss of the substrate origins from two parts, the mechanical wear by substrate-to-substrate contact and mild wear by tribofilm remove [26]. The mechanical wear $\left(h_{w}^{\text {mech }}\right)$ is regarded as adhesive wear and is derived from the direct substrate contact. It can be evaluated by Archard's wear model [12]:

$$
h_{w}^{\text {mech }}=\int K_{\text {sub }} u p_{\text {sub }} d t
$$

While the latter form of wear is caused by diffusion of the substrate atoms into the tribofilm, which are subsequently loss if the tribofilm is removed. The loss of the substrate material due to atom diffusion is calculated considering both the tribofilm remove rate and the concentration of the substrate material atoms in the tribofilm $[25,26]$. The concentration in the tribofilm is higher close to the substrate-tribofilm interface and reduces away from the interface. The loss of the substrate material (mild wear, $h_{w}^{\text {mild }}$ ) can then be calculated [13]:

$$
h_{w}^{\text {mild }}=\int e^{-c h_{t r i}}\left(\frac{\partial h_{t r i}}{\partial t}\right)_{r e m} d t
$$

where the term $e^{-c h_{t r i}}$ is the concentration of substrate material and $c$ is an unknown constant which needs be determined experimentally. In this paper, it is obtained from [13]. $\left(\frac{\partial h_{t r i}}{\partial t}\right)_{r e m}$ is the tribofilm remove rate and is determined in the following section.

\subsection{Tribochemistry reaction}

The tribofilm thickness is an essential variable in the layered contact model (section 2.1) to 
determine the contact portion of the tribofilm. The tribofilm is continuously worn and replenished during surface rubbing and the evolution of the tribofilm thickness stems from the balance of its growth and remove rates. The change rate $\left(\frac{\partial h_{t r i}}{\partial t}\right)$ in the tribofilm thickness is calculated by subtracting the remove rate from the growth rate:

$$
\frac{\partial h_{t r i}}{\partial t}=\left(\frac{\partial h_{t r i}}{\partial t}\right)_{g r o}-\left(\frac{\partial h_{t r i}}{\partial t}\right)_{r e m}
$$

where $\left(\frac{\partial h_{t r i}}{\partial t}\right)_{\text {gro }}$ and $\left(\frac{\partial h_{t r i}}{\partial t}\right)_{r e m}$ are the tribofilm growth and remove rate respectively. Eq. 11 also denotes the net growth rate of the tribofilm.

\subsubsection{Tribofilm growth}

The growth rate $\left(\left(\frac{\partial h_{t r i}}{\partial t}\right)_{\text {gro }}\right)$ of the ZDDP tribofilm is calculated based on Eq. 12, which is known as the stress-activated Arrhenius equation [7].

$$
\begin{gathered}
\left(\frac{\partial h_{t r i}}{\partial t}\right)_{g r o}=\Gamma_{0} \exp \left(-\frac{\Delta U_{\mathrm{act}}-\tau \cdot \Delta V_{\mathrm{act}}}{k_{B} T}\right) \\
\tau=\mu p_{\text {asp }}
\end{gathered}
$$

In this equation, $\Gamma_{0}$ is the pre-factor, $k_{B}$ is Boltzmann's constant, $T$ is the absolute temperature, $\Delta U_{a c t}$ is the internal activation energy (i.e., the energy barrier in the absence of stress), and $\Delta V_{a c t}$ is the activation volume. The values of these parameters are obtained from [13]. It is worth noting that $\tau$ in this equation is shear stress [4] and $\mu$ is the CoF.

The stress-activated Arrhenius equation was initially derived through a single asperity (an AFM tip) sliding contact experiment and subsequently applied in deterministic model in which the surface contact pressure of identical asperity is calculated $[13,15]$. In this study, a statistical model is used and in each mesh $\left(10 \times 10 \mu \mathrm{m}^{2}\right)$ the asperity contact pressure is averaged based on the statistical characteristics of the surface topography. Thus, the tribofilm thickness in each mesh is represented by a uniform value. This is practical and more efficient as the size of an engineering friction pair (like the piston ring and cylinder liner pair in ICEs) is in macroscopic scale and the computation cost of a deterministic model is quite high.

\subsubsection{Tribofilm remove}

It was observed in experiments that the remove rate would increase linearly with the growth of the tribofilm [27], which is attributed to the difference in the wear resistance between the bulk of the substrate (higher wear resistance) and the free surface (lower wear resistance). Based on Archard's wear model with a variable wear coefficient, the tribofilm remove rate $\left(\left(\frac{\partial h_{t r i}}{\partial t}\right)_{r e m}\right)$ is calculated as follows:

$$
\begin{aligned}
& \left(\frac{\partial h_{t r i}}{\partial t}\right)_{r e m}=K u p_{a s p} \\
& K=K_{s u b}\left(1+\alpha h_{t r i}\right)
\end{aligned}
$$

where $K$ is the remove rate (or wear rate) coefficient, $K_{\text {sub }}$ is the wear rate of the substrate, 
and $\alpha$ is a constant describing the durability change rate inside the tribofilm. They can be calibrated by experiments. Eq. 14 and 15 indicate that the tribofilm remove rate varies linearly with its thickness, and similar models were also used in [15, 22].

However, experiments also showed that the tribofilm would collapse under an extremely high contact pressure and a threshold pressure for the tribofilm to maintain a stable thickness exists [7, 28]. Above the threshold pressure, the tribofilm formation is not stable and the remove process is dominant over the growth process. In this situation, the result using the linear tribofilm remove rate model (eq. 14 and 15) may be far from the realistic. Based on eq. 14 and 15, the authors propose a modified tribofilm remove model and introduce an impact factor reflecting asperity contact state according to the experiments results. The details will be discussed in section 4.2.

\subsubsection{Mechanical properties of the tribofilm}

The tribofilm has different mechanical properties compared to the substrate, and several studies have focused on the assessment of these properties [29-31]. The mechanical properties of the tribofilm determine the contact pressure and then the growth rate. It is generally agreed that the hardness of the tribofilm varies with its height basically in a linear way. Therefore, as the tribofilm growths and removes, its hardness evolves along with its height. To model this variable hardness, the approach presented by Andersson et al. is used in the present study [32].

Regarding as the effect of temperature, it was found that the hardness and the Young's modulus of the ZDDP tribofilm closely depended on the temperature, especially under a high temperature $[33,34]$ (above $220^{\circ} \mathrm{C}$, the ZDDP tribofilm starts to degrade [35]). It was observed by experiments that the hardness of the ZDDP tribofilm would be lower at a higher temperature [33], and based on this, Bosman and Schipper [36] proposed a compensation factor for the hardness as a function of temperature. According to Ref. [13], the hardness of the tribofilm at a given temperature with a certain thickness can then be obtained by multiplying a compensation factor from [36]. The mechanical model is general reasonable as it follows the trends of the tribofilm hardness decay with film thickness and temperature. The Young's modulus of the tribofilm is related to its hardness, and after a threshold hardness, it shows a linear increase with a slope of 35 [36, 37].

Asperity interaction and fluid viscous shear can produce considerable heat and the temperature in the contact area will rise to some extent under boundary lubrication. Studies showed that flash temperature may play a role in promoting ZDDP tribofilm growth at high sliding speeds, but not at the low sliding speeds $[38,39]$. In the present study, the sliding speed is extremely low (an average speed of $0.04 \mathrm{~m} / \mathrm{s}$ in a stroke), and thus the temperature variation due to frictional heating is minor. Nevertheless, the temperature increase was evaluated using the method by Bosman and De Rooij [40] and the maximum was found to be less than $1{ }^{\circ} \mathrm{C}$. As a result, the flash temperature at the sliding interface has a marginal effect on the ZDDP tribofilm formation as well as the macroscopic friction and wear, and thus the temperature increase by friction is neglected in the current simulations.

\subsection{Computation flowchart}

The flowchart of the boundary lubrication model is shown in Fig. 2. The computation starts 
with inputting the initial parameters, including the mechanical properties, macroscale geometry, roughness, load, temperature and sliding velocity. Then the oil film thickness is determined by the profile and an assumed minimum film thickness. The oil film pressure and asperity contact pressure are obtained by solving the average Reynolds equation and the layered statistical contact model respectively. Initially, there is no the tribofilm $\left(h_{t r i}=0\right)$. After the calculation of contact pressure and shear stress, the tribofilm growth and remove rate are determined using Eqs. 12-15, respectively. The tribofilm thickness is then updated accordingly. Successively, it is used in the contact model as the thickness of the tribofilm layer. The mechanical properties are updated based on the new tribofilm thickness. Next, the convergence on load balance is checked. If the convergence criterion is not satisfied, adjust the minimum film thickness and the calculation repeats. Otherwise, the friction force and wear depth are obtained from Eqs. 5-10. The simulation time is then increased by a certain time step and the whole computation will stop when reaches the set time.

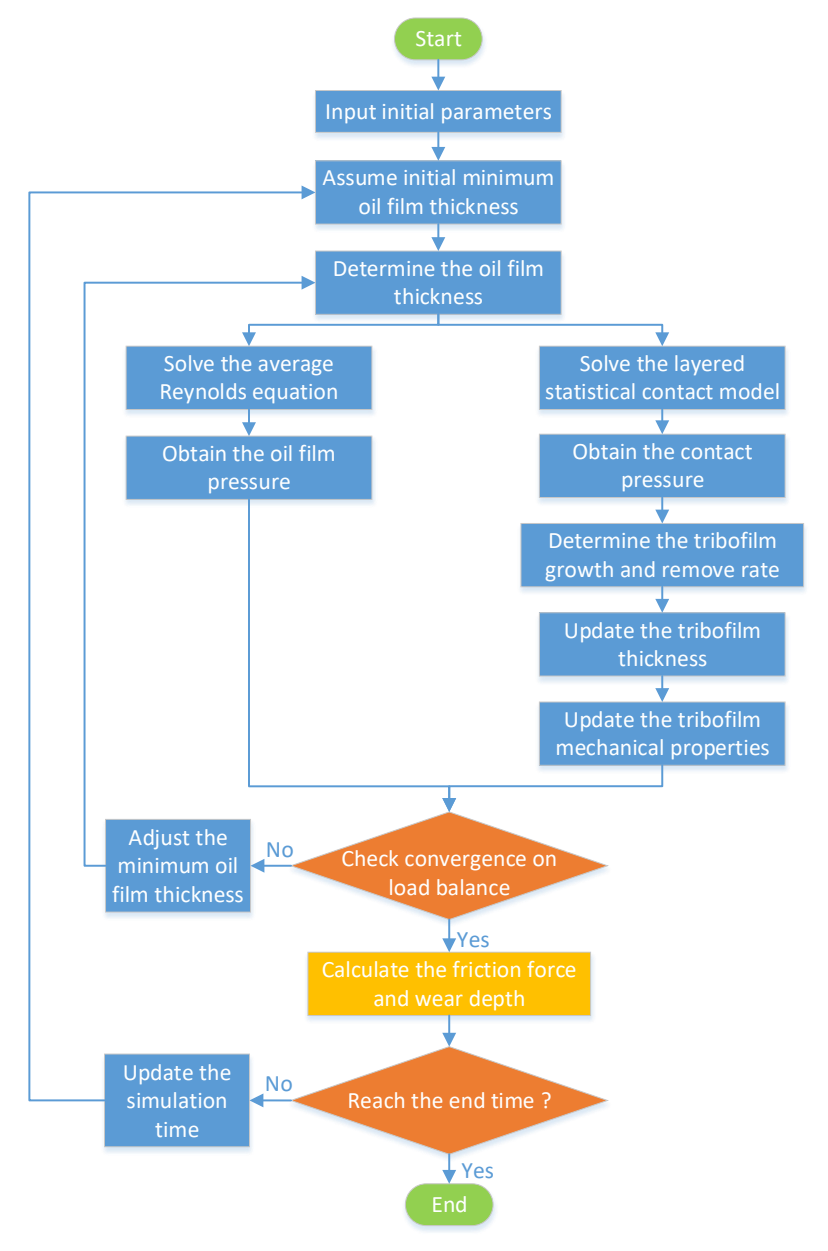

Fig. 2 Flowchart of the computation procedures for the boundary lubrication model.

\section{Boundary lubrication experiment}

The parameters in the boundary lubrication model need to be calibrated according to the experiment results. Successively the tribofilm thickness, CoF, and wear depth predicted by the 
model are compared with the ones measured in the reciprocating experiments under various lubricant temperatures and applied loads.

\subsection{Test materials}

The piston ring and cylinder liner segments are selected to be the test materials. They simulate a piston ring and cylinder liner (PRCL) contact, which usually experiences boundary lubrication state near the TDC and BTC in ICEs. In this study, the piston ring segments are cut off from a top compression ring with a diameter of $110 \mathrm{~cm}$. The ring working surface has diamond-like carbon (DLC) coating. Spheroidal graphite cast cylinder liner in an ICE, which is mating with the ring, is sectioned into liner samples with a size of $15 \times 10 \times 5 \mathrm{~mm}^{3}$. Surface roughness measurements are performed by Mitutoyo CV-4500S4 surface profiler over a data length of $4 \mathrm{~mm}$ and $l c$ filter cut off length of $0.8 \mathrm{~mm}$. The mechanical properties and surface roughness of the piston ring and liner samples used in this study are listed in Tab. 1.

To simulate the lubrication environment in engineering friction pairs more realistically, an industrial lubricant, SAE 10W40, which is a ZDDP-fortified mineral engine oil for classic cars, is used in the present study. The properties of the lubricant are shown in Tab. 2.

Tab. 1 Mechanical properties and surface roughness parameters of the piston ring and liner samples.

\begin{tabular}{ccc}
\hline Label & Piston ring & Liner \\
\hline Young's modulus, $E(\mathrm{GPa})$ & 155 & 205 \\
Hardness $(\mathrm{HV})$ & 2000 & 370 \\
Poisson's ratio & 0.3 & 0.3 \\
Surface roughness, $\sigma(\mu \mathrm{m})$ & 0.416 & 0.627 \\
Asperity radius, $\beta(\mu \mathrm{m})$ & 7.46 & 7.57 \\
Asperity density, $\eta\left(\mu \mathrm{m}^{-2}\right)$ & 0.052 & 0.038 \\
\hline
\end{tabular}

Tab. 2 Properties of the test lubricant.

\begin{tabular}{cc}
\hline Label & Value \\
\hline Viscosity, kinematic at $40{ }^{\circ} \mathrm{C}$ & $100.9 \mathrm{cSt}$ \\
Viscosity, kinematic at $100{ }^{\circ} \mathrm{C}$ & $15.1 \mathrm{cSt}$ \\
Viscosity index & 157 \\
Density at $20{ }^{\circ} \mathrm{C}$ & $0.861 \mathrm{~kg} / \mathrm{l}$ \\
Flash point & $220^{\circ} \mathrm{C}$ \\
Zinc & $0.14 \mathrm{wt} \%$ \\
Phosphorus & $0.12 \mathrm{wt} \%$ \\
\hline
\end{tabular}




\subsection{Experimental rig}

The experiments are conducted using a reciprocating tribometer (MFT-R4000). The tribometer is shown in Fig. 3(a) and (b), and a schematic diagram of the ring-on-liner contact is shown in Fig. $3(\mathrm{c})$. The ring segment is oscillating against the liner segment with a stroke length of $10 \mathrm{~mm}$ while the latter is fixed in the oil bath. A conformal ring holder is used to prevent any inclination to the counter-body. The reciprocating frequency is as low as $2 \mathrm{~Hz}$ to ensure the boundary lubrication appears in a stroke, which resulting in an average speed of $0.04 \mathrm{~m} / \mathrm{s}$. Before each test, the oil bath is filled with lubricant, which completely covers the piston ring, i.e. the piston ring is under fully flooded lubrication. The lubricant temperature is set by the heating system under the oil bath from ambient temperature to $200{ }^{\circ} \mathrm{C}$. The maximum applied load is up to $300 \mathrm{~N}$. In this study, the duration time of each test is $2 \mathrm{~h}$. The $\mathrm{CoF}$, the test liner temperature (measured by a thermocouple at the bottom of the liner segment), the applied load, and the stroke are continuously monitored and recorded by the tribometer software.

Before each test starts, a 30-minute running-in is conducted under the load of $20 \mathrm{~N}$ and the temperature of $30{ }^{\circ} \mathrm{C}$. Continually the load and temperature are increased to the set values. Each test is repeated three times to ensure consistency of the results.

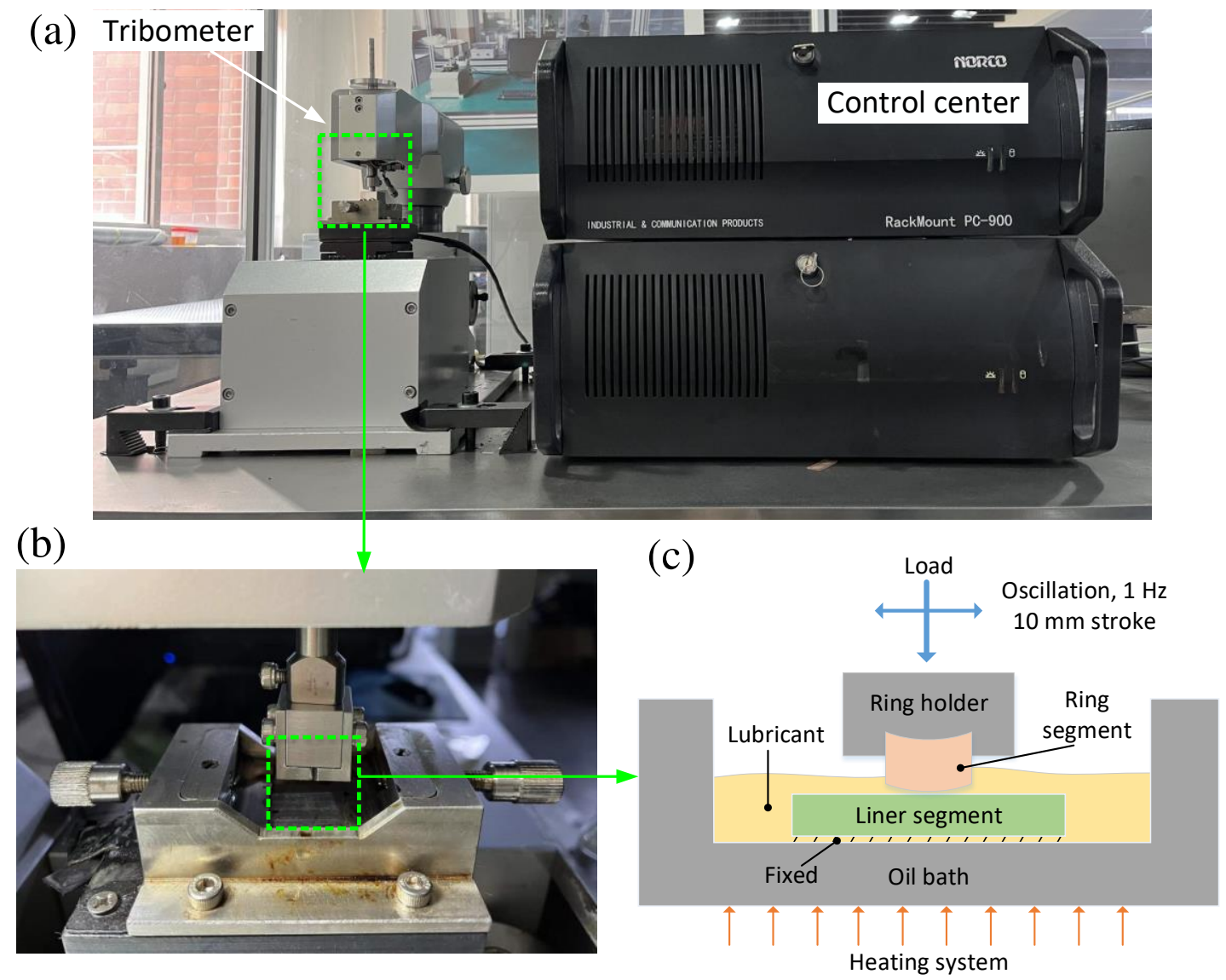

Fig. 3 Tribo-test set-up used for the present study $(a, b)$ reciprocating tribometer (MFT-R4000), and (c) schematic principle of the ring-on-liner contact. 


\subsection{Surface characterization and tribofilm measurement}

The wear depth of the liner segment is measured using the surface profiler. Measurements are taken from multiple locations across the wear track to determine standard deviation and the final wear depth is the average of these measurements. The surfaces after the experiments are observed without any conductive coating by the scanning electron microscope (SEM) operating under high vacuum. The chemical composition analyses are carried out by energy dispersive X-ray spectroscopy (EDX) and quantitative analysis of the EDX spectra is performed using the Aztec software.

In previous studies, the tribofilm thickness can be mapped in situ by spacer layer interferometric method cooperated with mini traction machine (MTM) ball-on-disc rig [41] or scanned ex situ by atomic force microscopy (AFM) [42]. The surface roughness of the tested samples in these studies is as low as dozens of nanometers or even lower. Yet in the present study, the surface roughness of the tested liner segment is much higher (hundreds of nanometers). It would be very difficult to measure the tribofilm thickness using these two methods aforementioned.

The resolution in the height direction of a 3D laser-scanning confocal microscope (Keyence VK-X100/X200) is sub nanometer and its measurement area can be expanded to millimeters. Therefore, it is suitable for surface topology measurement and tribofilm thickness evaluation in the present study. It has been reported that ethylenediaminetetraacetic acid (EDTA) can dissolve the ZDDP tribofilm without damaging the substrate material [43]. After each test, the liner segment is carefully cleaned using anhydrous ethanol. A texture dimple is then marked within the wear scar on the liner surface by a high accurate picosecond laser machine (Trumpf, TruMicro 5050, German). The depth of the texture dimple is micrometer scale and it completely penetrates the tribofilm reaching the substrate material. Subsequently, the same region including the texture dimple is measured using the confocal microscope twice: (i) before removing the ZDDP tribofilm; (ii) after removing the ZDDP tribofilm with $0.05 \mathrm{~mol} / \mathrm{L}$ EDTA disodium salt in distilled water solution. The above procedure is repeated twice in different regions in the central area of the wear scar for each liner. The tribofilm distribution and thickness are then obtained by subtracting the latter measurement results from the former (see Fig. 6). Similar tribofilm measurement method was used in [44].

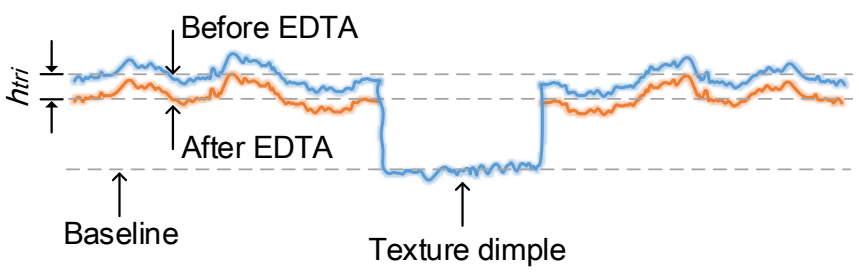

Fig. 4 The schematic principle of tribofilm measurement.

\section{Results and discussion}

In the boundary lubrication model, there are some key parameters that require calibration 
before predictions of surface wear and friction force can be made. They are determined using experimental results for a given operating condition and then used for all other simulations under different conditions. The calibration experiment is conducted under the lubricant temperature of 90 ${ }^{\circ} \mathrm{C}$ and load of $160 \mathrm{~N}$. The CoF of the substrate is obtained by dry sliding tribotest and here it is 0.15 for the present test samples. As for the CoF of the ZDDP tribofilm, it is determined by fitting the simulated $\mathrm{CoF}$ with the recorded $\mathrm{CoF}$ by the calibration experiment, and it is 0.11 . In the tribofilm remove and substrate wear model, $K_{\text {sub }}$ (the wear rate of the substrate) and $\alpha$ are calibrated based on the wear depth and tribofilm thickness measurements. In the present study, $\alpha$ equals to $0.21 \mathrm{~nm}^{-1}$ and $K_{\text {sub }}$ is $1.43 \times 10^{-16} \mathrm{~Pa}^{-1}$.

The simulation results below are obtained using the calibrated boundary lubrication model. Fig. 5(a) shows the sliding velocity of the reciprocating tribometer in one stroke (red line) and the maximum velocity is $0.064 \mathrm{~m} / \mathrm{s}$. The oil film thickness ratio, which equals to $h / \sigma$, represents the lubrication state and is also predicted in one stroke (blue dash line) under the lubricant temperature of $100{ }^{\circ} \mathrm{C}$ and load of $150 \mathrm{~N}$. Its maximum value is 0.18 and is much lower than the threshold of boundary lubrications. Fig. 5(b) shows the experiment and simulation results during a reciprocating sliding test under the same operating condition. The CoFs recorded by the tribometer and predicted by the boundary lubrication model exhibit a consistent tendency with the sliding time: they both decline rapidly initially and then remain stable. This is different from the results of previous ballon-disk experiments, like $[45,46]$. It is explained as follows. The surface roughness of the test specimen (ball and disk) is only dozens of nanometers, while the tribofilm thickness generated on the specimen surface can exceed one hundred nanometers during rubbing. It can substantially reshape the topography of the initial rubbing surfaces and increase the roughness significantly. Successively, the oil fluid entrainment is inhibited and asperity contact friction increases [46]. However, in the present study, the surface roughness of the test ring and liner samples is as high as hundreds of nanometers. The formation of ZDDP tribofilm has marginal effect on the roughness of the rubbing surfaces. On the contrary, the CoF of the tribofilm (0.11) is lower than the one of the substrate material (0.15). Thus the total friction will decrease (Fig. 5(b), red and blue dash lines) as the tribofilm grows (Fig. 5(b), green dash line). At the same time, the applied load will be more born by the tribofilm with a higher thickness according to the layered statistical contact model (section 2.1). The part born by the oil film will be lighter, which results in the oil film thickness being lifted slightly (Fig. 5(b), orange dash line). Azam et al. [47] simulated the effects of tribofilms on lubrication. They found that the presence of tribofilm increased the lubricant film thickness and also improved the lubrication regime by helping entrain more lubricant inside the contact area. 

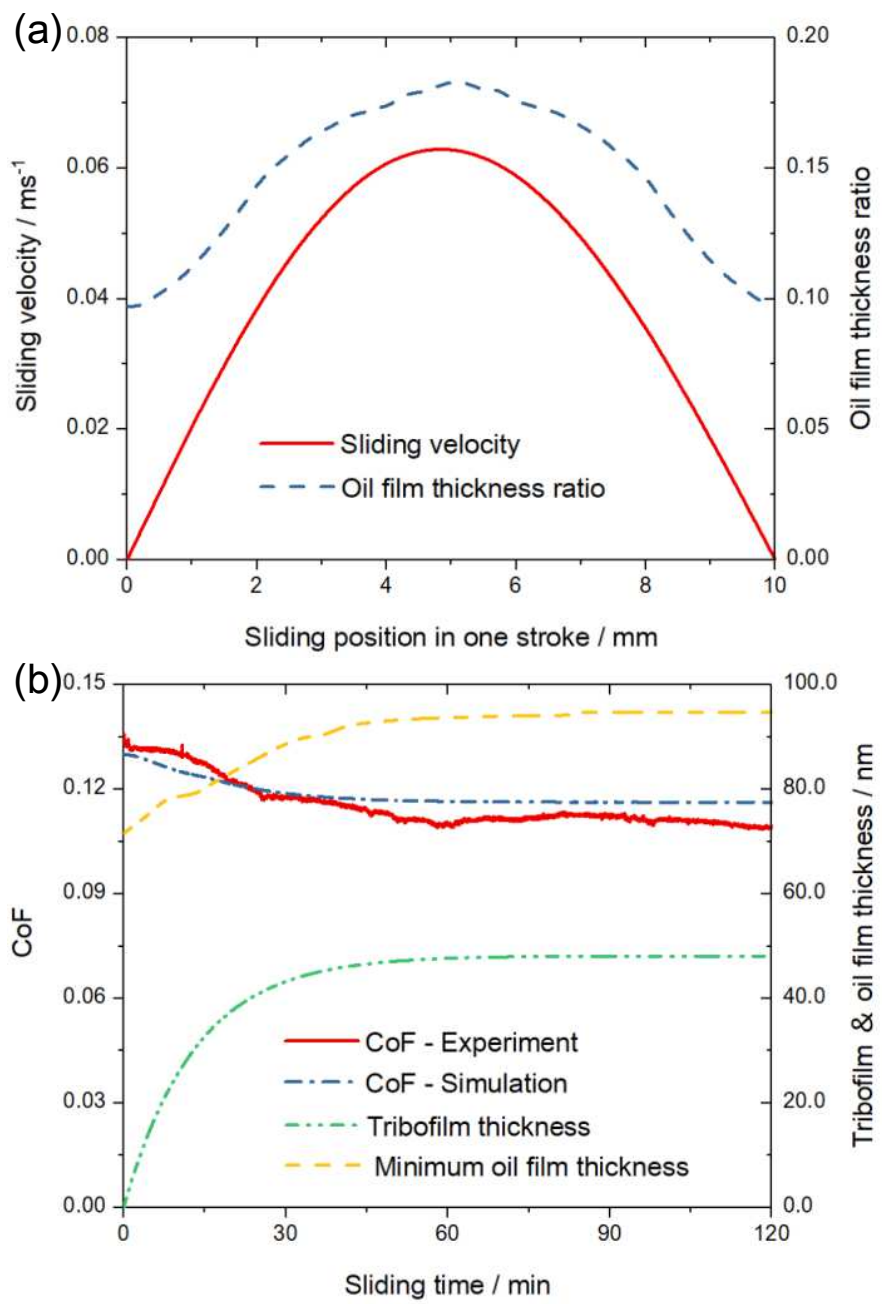

Fig. 5 (a) sliding velocity and oil film thickness ratio in one stroke and (b) experiment and simulation results during a representative reciprocating sliding test.

The results from SEM imaging and EDX spectra are shown in Fig. 6. The SEM image clearly shows wear tracks parallel to the sliding direction (S.D.) on the surface of the liner segment. As typical elements of ZDDP molecular, the enrichment of zinc and phosphorus (in EDX chemical maps) on the wear track indicates the formation of corresponding poly-phosphate tribofilm. Note that higher concentrations of zinc and phosphorus appearing in the wear tracks means a thicker tribofilm, which is also observed in previous research using Raman spectroscopy [44]. It demonstrates that the tribofilm mainly forms in the wear track area, where the contact pressure and shear stress are much higher. According to the stress-activated tribochemistry theory, the tribofilm growth will be promoted in these areas. Similar element distributions where zinc and phosphorus are concentrated in the wear tracks were obtained in the ball-on-disk reciprocating experiments [48]. High magnification SEM image and EDX spectra (the bottom panels of Fig. 6) confirm that the enrichment of zinc and phosphorus emerges in the wear track. 

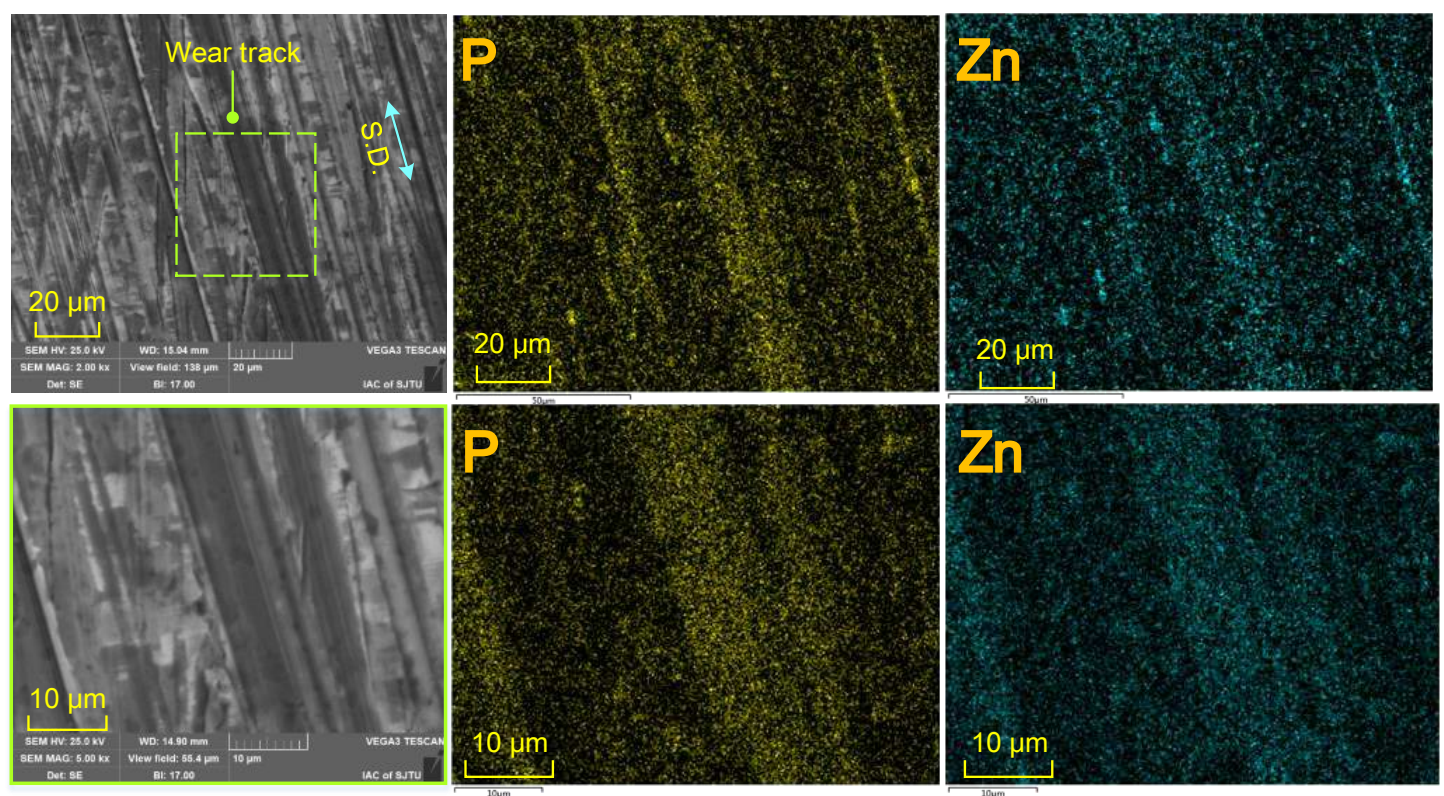

Fig. 6 Representative SEM images and EDX maps of $\mathrm{P}$ and $\mathrm{Zn}$ demonstrating a tribofilm generated on the liner segment from the reciprocating tribometer.

As mentioned in section 3.3, the tribofilm measurement is using the 3D laser-scanning confocal microscope and calibrated by a texture dimple. The 3D surface topographies pre-EDTA and postEDTA scanned by the confocal microscope are set on the same baseline under the same magnification scale. Then their gap in the height direction can be obtained by subtracting the latter measurement results from the former, representing the average ZDDP tribofilm thickness (Fig. 4). The baseline is defined by the bottom of the texture dimple with a diameter of $150 \mu \mathrm{m}$ marked within the wear scar. To avoid the complex metal oxidation and sublimation area by lase ablation, the tribofilm thickness evaluation areas $\left(150 \times 150 \mu \mathrm{m}^{2}\right)$ are chosen away from the texture dimple in its four directions (seen Fig. 7(a)). Fig. 7(b) displays the cross-sectional profile of the texture dimple by the surface profiler. On the one hand, the depth of the texture dimple is about $1.8 \mu \mathrm{m}$, and it completely penetrates the tribofilm layer reaching the substrate material. Therefore the EDTA treatment won't change the topography of the dimple bottom. On the other hand, the bottom profile is relative smooth and quite flat owing to the high-accuracy picosecond laser machine. It guarantees the reliability of the dimple bottom to be the baseline. The measurement method described above is practical as the ZDDP tribofilm is uneven and not continuous. The measurement results of the tribofilm thickness under various temperatures and loads will be shown and discussed below. 
(a)
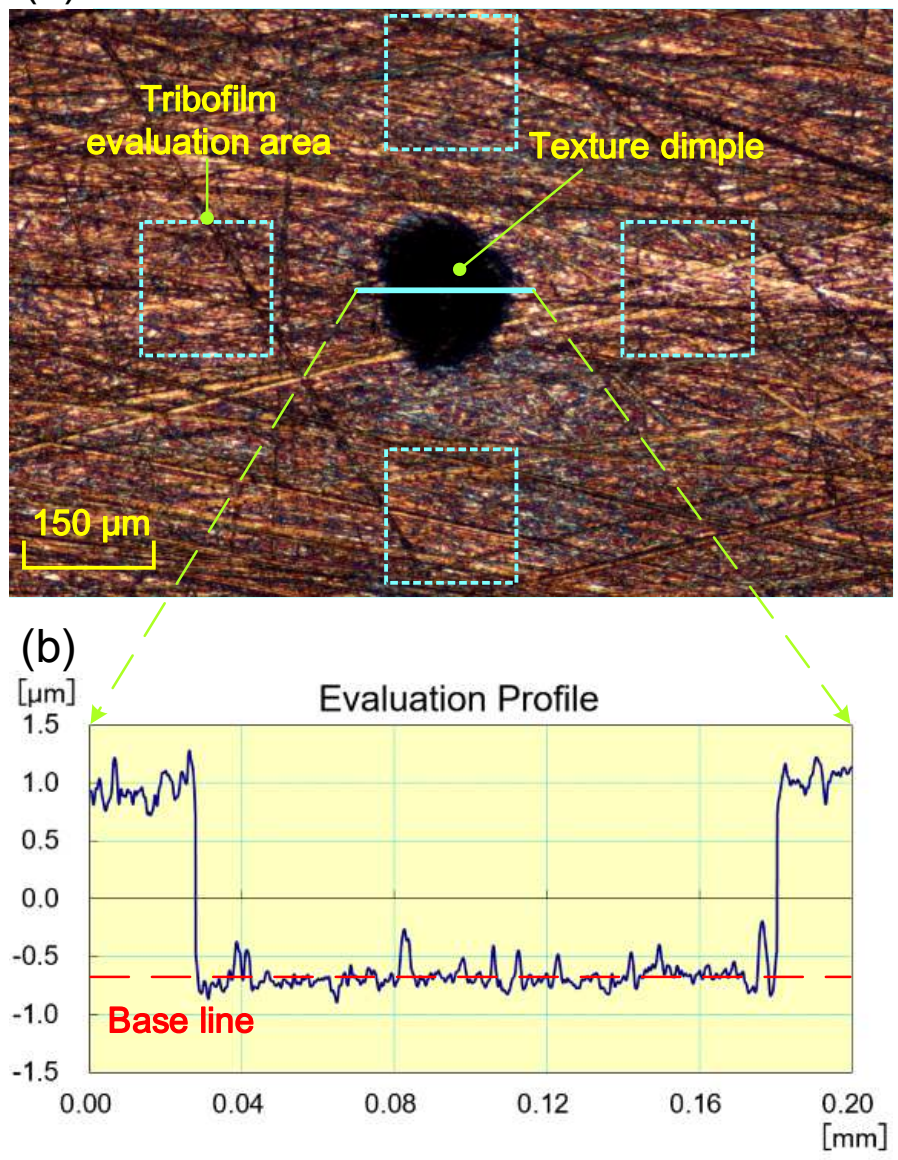

Fig. 7 The texture mark in the wear scar on the liner surface: (a) the optical microscope image of the texture dimple and tribofilm measurement areas (b) the texture cross-sectional profile.

\subsection{Effect of lubricant temperature}

The tests are firstly conducted over a wide range of lubricant temperatures under the load of 150 N. Fig. 8 summarizes the tribofilm measurement areas (marked by cyan dash squares in Fig. 7) before and after EDTA treatment under the lubricant temperatures from 40 to $140{ }^{\circ} \mathrm{C}$. These focal microscope images clearly demonstrate that the ZDDP tribofilm has been entirely removed from the surface by EDTA treatment and the substrate material is exposed. The quantitative tribofilm thickness values are plotted as a function of temperature in Fig. 9. As the lubricant temperature rises, the tribofilm thickness increases rapidly and basically in an exponential way. The predicted tribofilm thickness by the boundary lubrication model agrees well with the experimental data, which again confirms the validity of the stress-augmented thermal activation model. This trend is similar to the previous works observed in ball-on-disk experiments $[4,8]$ and simulated by a deterministic tribofilm growth model [13]. It is noticeable that the topographies of the tribofilm shown in Fig. 9 display a strip-like distribution parallel to the sliding direction. It is reasonable as the tribofilm mainly grows in the wear track area and it also matches the EDX map shown in Fig. 6. The tribofilm thickness results demonstrate that the temperature can evidently promote the tribo-chemistry reaction forming ZDDP tribofilm. 

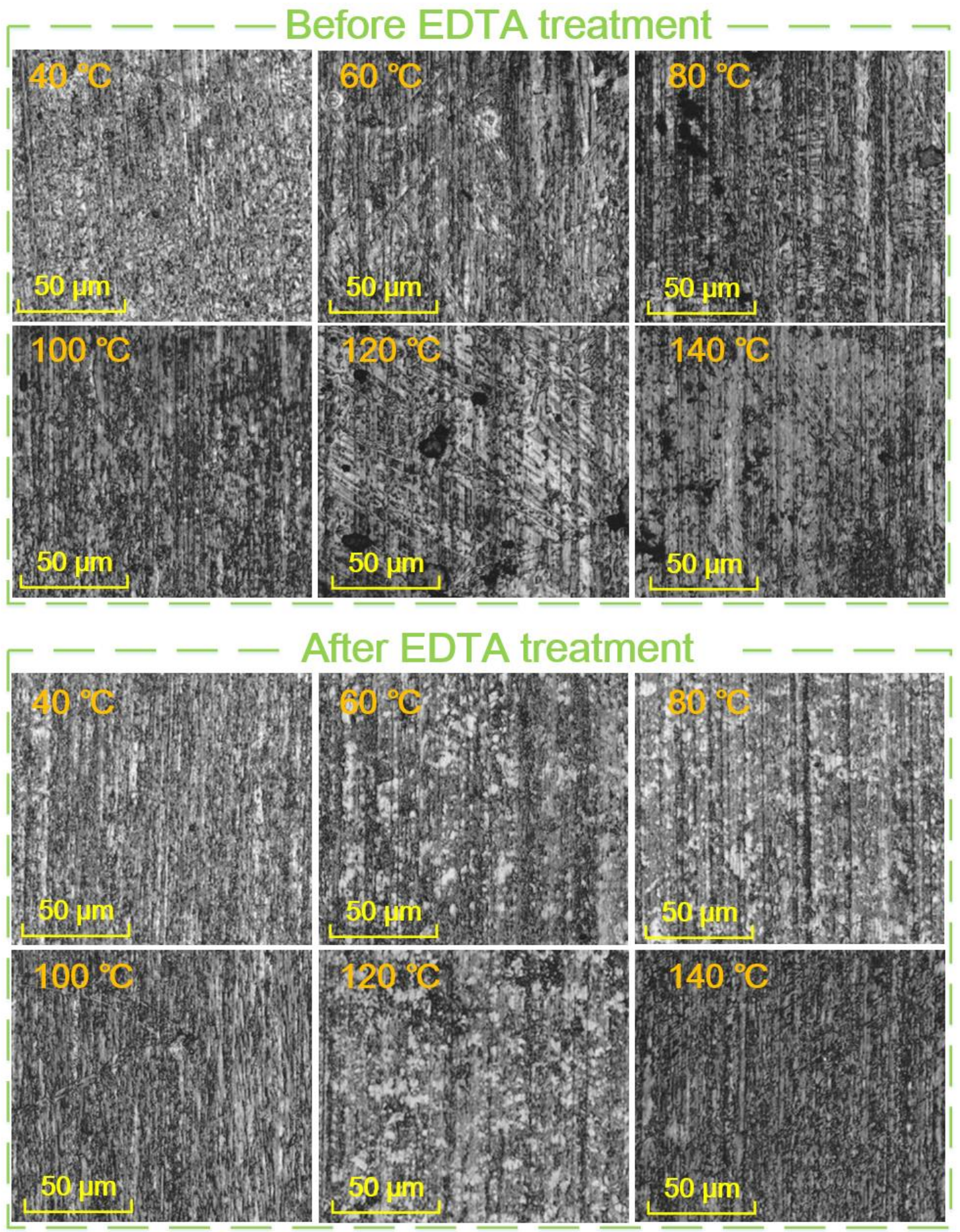

Fig. 8 Confocal microscope images of the liner segments under various temperatures: before and after EDTA treatment. 


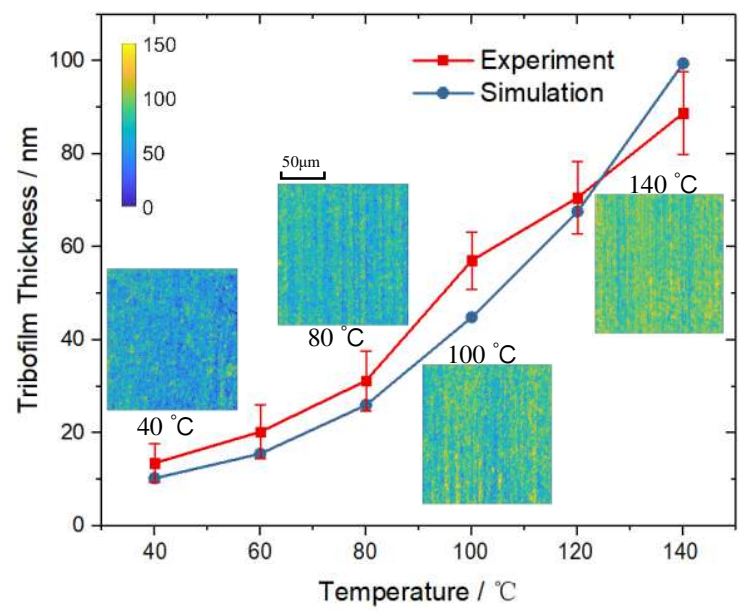

Fig. 9 Tribofilm thickness results under various temperatures: experiment vs. simulation.

To further study the effect of lubricant temperature on the friction and wear under boundary lubrication, the CoFs and wear depths measured in experiments and predicted by simulations with and without tribofilm are compared in Fig. 10. Here simulations without tribofilm means that the stress-augmented thermal activation model is not included and the tribofilm formation is not considered in the simulations. Therefore there are only lubricant fluid pressure and substrate contact pressure to support the applied load and the surface wear merely stems from mechanical interaction. It is in fact a mixed lubrication model. As shown in Fig. 10 (green line chart), the CoFs predicted by the model without tribofilm tends to rise with the increasing temperature. This is easy to understand as the lubricant viscosity will decline when the temperature rises, which leads to a thinner oil film. Then more asperities come into contact. As a result, the friction force increases. However, the CoFs recorded by the tribometer (red line chart) in the same temperature range shows an opposite tendency: it declines with the increasing temperature. When the growth of tribofilm is included in the lubrication model, the simulated CoFs (blue line chart) matches the experimental data well. It is reasonable as the tribofilm thickness increases with the rising temperature (Fig. 9), and according to the layered statistical contact model (section 2.1), the tribofilm will share more applied load. Due to its low friction characteristics, the total friction force calculated by Eq. 5 will decrease. In the ball-on-disk experiments by Xu et al. [44], the tribofilm formation rate was also promoted under a higher lubricant temperature followed by a decreased CoF. The inverse trends presented by the simulations with and without tribofilm emphasize the difference between the boundary and mixed lubrication.

Analogously, the wear depths (Fig. 10, histogram charts) predicted by the model excluded tribofilm becomes larger under a higher lubricant temperature owing to the severer contact state. When the tribofilm growth model is integrated in the simulations, the wear depths decrease with the rising temperature, which is also consistent with the experiment data. As aforementioned in section 2.5 , here the wear in the present model comprises two parts: the mild wear and the mechanical wear. The mild wear originates from the diffusion of the substrate material atoms into the tribofilm and subsequently lost by tribofilm remove [36]. The atom concentration of substrate material substantially determines this kind of wear (Eq. 10) and it is reducing with the increasing tribofilm thickness. The mild wear will therefore decrease with the increasing temperature as a thicker tribofilm is generated. In the simulations by Akchurin et al. [13] as well as by Ghanbarzadeh et al. 
[11], the predicted mild wear was also decreasing under a higher lubricant temperature. For all that, the authors would like to point out that in the boundary lubrication experiments [11], only mild wear was considered, yet substrate contact and mechanical wear would inevitably occur noting the tribofilm is highly uneven and patchy. Apart from the mild wear, the mechanical wear also displays a negative relation to the temperature shown in Fig. 10. The reduction in the mechanical wear is attributed to the formation of the protective tribofilm film, which avoids the direct contact and effectively inhibits the mechanical wear of substrate materials. The wear resistance of the ZDDP tribofilm is more significant under a higher lubricant temperature, which is also consistent with the simulation results by Chen et al. [15].

The simulation analysis above can properly explain the experiment results and it demonstrate that the model proposed in the present paper is able to capture the friction and wear behaviors of the boundary lubrication.

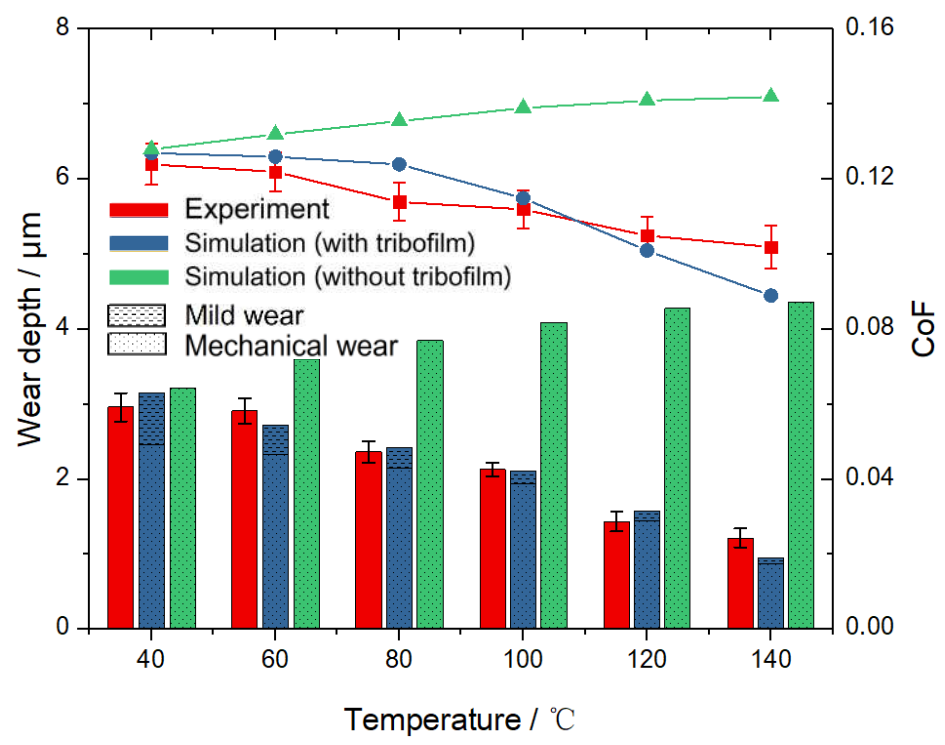

Fig. 10 CoFs (line charts) and wear depths (histogram charts) under various temperatures: experiment vs. simulation.

\subsection{Effect of applied load}

Contact pressure can enhance the growth of the ZDDP tribofilm, while studies have also shown that the tribofilm wear/remove will be dominant if the pressure is too high [7]. The final tribofilm thickness depends on the equilibrium between the tribofilm growth and removal rate [49]. To study the effect of the contact pressure and shear stress, tests were then conducted over a wide range of applied loads under the lubricant temperature of $100{ }^{\circ} \mathrm{C}$. The confocal microscope images of the tribofilm evaluation areas before and after EDTA treatment from 50 to $300 \mathrm{~N}$ are summarized in Fig. 11. The quantitative tribofilm thickness values are plotted as a function of load in Fig. 12 and similar strip-like distributions of the tribofilm topography are also displayed. The experiment data (red line chart) shows that as the applied load goes up, the tribofilm thickness will be increasing first but declines under the maximum load. It is supposed that a moderate applied load will boost the tribofilm growth but a much higher load will depress its formation by augmenting the tribofilm remove rate. In the single AFM tip sliding experiments by Gosvami et al. [7], they observed an 
unstable growth rate when the contact pressure exceeded 5.2 GPa. Bayat et al. [28] pointed out that there may be a threshold pressure for the tribofilm to maintain a stable thickness. Above this threshold pressure, the tribofilm formation is depressed and the remove is dominant. Yet the threshold pressure is sensitive to the surface roughness and thus hard to determine. In the present study, the authors initially used a linear tribofilm remove model (Eq. 14 and 15) based on the Archard's wear model whose remove rate coefficient varies with the tribofilm thickness. The predicted tribofilm thickness using this model agrees well with the experiment results under the moderate applied loads. However when the applied load exceeds $250 \mathrm{~N}$, the gap between the simulation and the experiment is unbearable. Considering the double-edge effect of the contact pressure, the authors proposed a modified tribofilm remove model by introducing an impact factor reflecting asperity contact state to the remover rate coefficient $K$. Specifically, the present model introduced a nonlinear pressure dependence to Eq. 15 by means of a multiplication factor, as shown in Eq. 16.

$$
K=K_{\text {sub }}\left(1+\alpha h_{\text {tri }}\right)\left[\beta\left(\frac{p}{p_{0}}\right)^{\gamma}+1\right]
$$

Here $\left[\beta\left(\frac{p}{p_{0}}\right)^{\gamma}+1\right]$ is the multiplication factor and is highly nonlinear with the contact pressure. $\beta$ and $\gamma$ are constants and can be calibrated according to experiments. In the present study, they are 24.86 and 11.96 , respectively. $p_{0}$ is the contact pressure when the nominal distance between the two rough surfaces is zero, that is, $h=0$. Note that the minimum values of $\left[\beta\left(\frac{p}{p_{0}}\right)^{\gamma}+1\right]$ equals to 1 when $p$ is 0 and its maximum is $\beta+1$. Under a light load condition where the contact state is mild, the tribofilm remove rate yielded by the modified model is quite close to the one by the linear model. While the former will be significantly higher than the latter by dozens of times under a severe contact state. As show in Fig. 12, the predicted tribofilm thickness by the modified model is in line with the experimental data in the whole range of the applied load. 

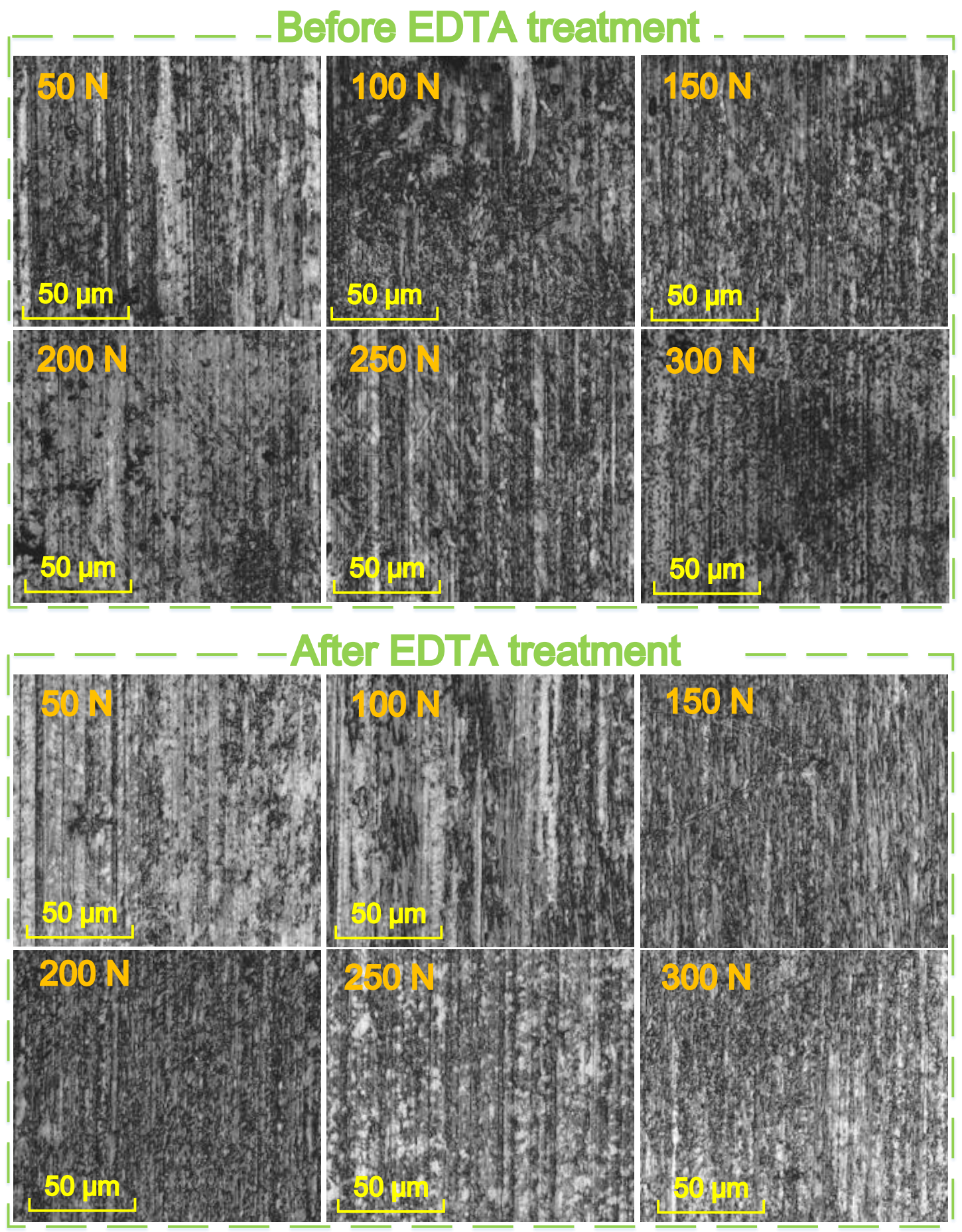

Fig. 11 Confocal microscope images of liner segments under various loads: before and after EDTA treatment. 


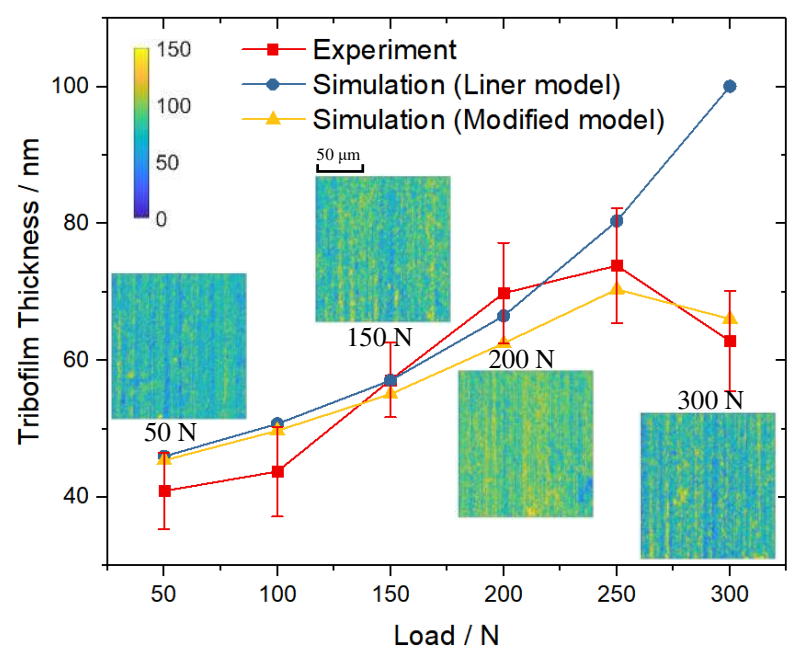

Fig. 12 Tribofilm thickness results under various loads: experiment vs. simulation.

To further examine the modified tribofilm remove model, the CoFs and wear depths predicted by the linear and modified model are both shown. They are compared with the measured results in the experiments and discussed below. The measured CoFs (Fig. 13, red line chart) declines first following the increasing applied load and reaches its minimum values about 0.106 . Then it is increasing as the load becomes higher. The simulation results using the linear remove model (blue line chart) keep decreasing along with the increasing load and are getting deviated from the experiment when the load exceeds $250 \mathrm{~N}$, just like the tribofilm thickness results in Fig. 12. Using the modified model, the simulation results (orange line chart) can catch the variation of the experiment results. The authors propose that the rise of $\mathrm{CoF}$ under the high applied load may result from the decrease of tribofilm thickness, comparing the results of the linear and modified model. The wear depths no matter by simulations or experiments are increasing with the applied load. For the simulation results, the mild wear only counts a small portion and it is the mechanical wear that mainly contributes to the total wear increase. It can be concluded from Eq. 15 that although the remove rate is increased under a higher load, the concentration of substrate material is also lower as the tribofilm increases (Fig. 12). Therefore the mild wear changes slightly. It is noteworthy that when the load increases from $250 \mathrm{~N}$ to $300 \mathrm{~N}$, the experimental wear depth rises significantly, which is also matches the variation of CoF. The simulation using the modified model shows a similar tendency with the experiment result.

The results and analysis above demonstrate that the linear tribofilm remove model may not suit for the severe contact state, while the modified model shows a favorable adaptation under a wide range of applied load. 


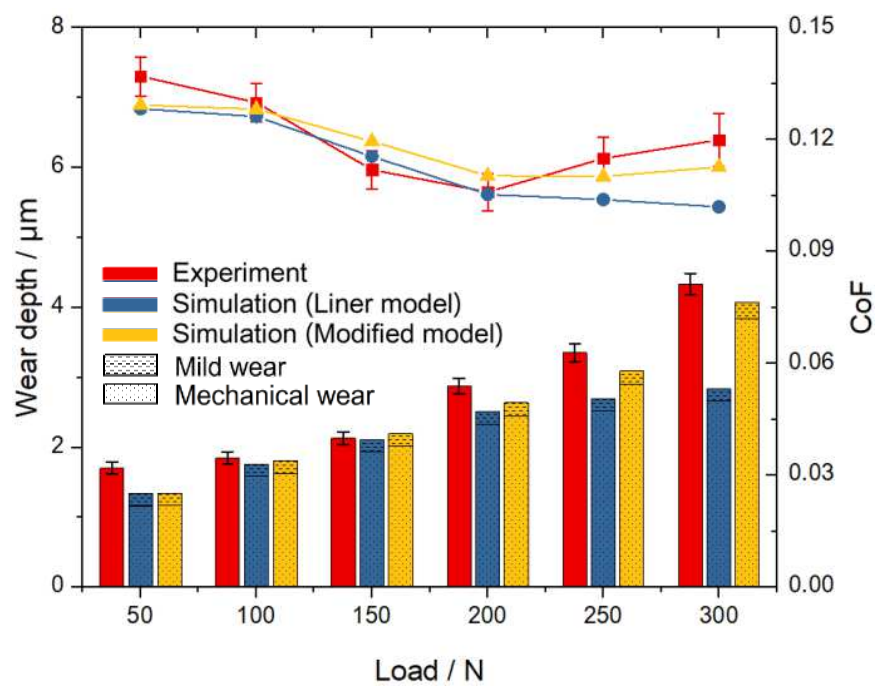

Fig. $13 \mathrm{CoFs}$ (line charts) and wear depths (histogram charts) under various loads: experiment vs. simulation.

\section{Conclusions}

A boundary lubrication model considering the ZDDP tribofilm growth and remove is developed, in which the asperity contact pressure is calculated using a layered statistical contact model. The simulated tribofilm thickness, friction and surface wear are compared with the reciprocating experiments under various temperatures and loads. The results can be summarized as follows:

1. The ZDDP tribofilm growth will be promoted under a high temperature and its thickness increases basically in an exponential way. As the applied load becomes heavier, the tribofilm will increase first, yet it will decrease if the load is too high due to the dominant tribofilm remove process. To catch the variation of the tribofilm thickness under the severe contact state, a modified remove model is proposed and it fits the experiment results well.

2. In the present study, as the tribofilm grows, the friction force will decline attributing to the lower $\mathrm{CoF}$ of the tribofilm comparing with the $\mathrm{CoF}$ of the substrate. The friction force shows an decreasing tendency with the rising temperature from 40 to $140{ }^{\circ} \mathrm{C}$, which are opposite to the simulation results without tribofilms.

3. The wear depth keeps decreasing with the rising temperature, and the effect on wear resistance of the ZDDP tribofilm reaches its maximum under the highest lubricant temperature. On the contrary, the wear depth will be higher under a heavier applied load, which is mainly attributed to the mechanical wear as the mild wear only counts a small portion of the total wear.

4. The simulation results of $\mathrm{CoF}$ and wear depth using the modified tribofilm remove model are well agreed with the experiments, which demonstrates that the present boundary lubrication model is validate. 


\section{Acknowledgement}

This study was supported by the National Natural Science Foundation of China (52130502, 51875344) and the stable support project for basic military research institutes (WDZC-2019-JGKK$03)$.

\section{Declarations}

Funding This study was supported by the National Natural Science Foundation of China (52130502, 51875344) and the stable support project for basic military research institutes (WDZC-2019-JGKK$03)$.

Author Contributions Conceptualization, B.L., L.Z., and X.M.; Data curation, B.L., L.Z., and X.M.; Formal analysis, B.L.; Funding acquisition, X.M.; Investigation, B.L. and L.Z.; Experiment, B.L.; Software, B.L.; Supervision, X.M. and C.W. All authors have read and agreed to the published version of the manuscript.

Conflict of interest The authors declare no potential conflicts of interest with respect to the research, authorship and/or publication of this article, and we have fully respected the research ethics principles.

\section{References}

[1] Zhang, J., Meng, Y. Boundary lubrication by adsorption film. Friction 3:115-147 (2015).

[2] Lyu, B., Meng, X., Zhang, R., Wen, C. A deterministic contact evolution and scuffing failure analysis considering lubrication deterioration due to temperature rise under heavy loads. Engineering Failure Analysis 123:105276 (2021).

[3] Spikes, H. The history and mechanisms of ZDDP. Tribology letters 17:469-489 (2004).

[4] Zhang, J., Spikes, H. On the mechanism of ZDDP antiwear film formation. Tribology Letters 63:115 (2016).

[5] Fujita, H., Spikes, H.A. The formation of zinc dithiophosphate antiwear films. Proceedings of the Institution of Mechanical Engineers, Part J: Journal of Engineering Tribology 218:265-278 (2004).

[6] Westerfield, C., Agnew, S. IR study of the chemistry of boundary lubrication with high temperature and high pressure shear. Wear 181:805-809 (1995).

[7] Gosvami, N., Bares, J., Mangolini, F., Konicek, A., Yablon, D., Carpick, R. Mechanisms of antiwear tribofilm growth revealed in situ by single-asperity sliding contacts. Science 348:102-106 (2015).

[8] Zhang, J., Ewen, J.P., Ueda, M., Wong, J.S., Spikes, H.A. Mechanochemistry of zinc dialkyldithiophosphate on steel surfaces under elastohydrodynamic lubrication conditions. ACS applied materials \& interfaces 12:6662-6676 (2020).

[9] Zhang, J., Ueda, M., Campen, S., Spikes, H. Boundary Friction of ZDDP Tribofilms. Tribology Letters 69:1-17 (2021).

[10] Ueda, M., Spikes, H., Kadiric, A. In-situ observations of the effect of the ZDDP tribofilm growth 
on micropitting. Tribology International 138:342-352 (2019).

[11] Ghanbarzadeh, A., Parsaeian, P., Morina, A., Wilson, M.C., van Eijk, M.C., Nedelcu, I., et al. A semi-deterministic wear model considering the effect of zinc dialkyl dithiophosphate tribofilm. Tribology Letters 61:1-15 (2016).

[12] Archard, J. Contact and rubbing of flat surfaces. Journal of applied physics 24:981-988 (1953).

[13] Akchurin, A., Bosman, R. A deterministic stress-activated model for tribo-film growth and wear simulation. Tribology letters 65:59 (2017).

[14] Azam, A., Ghanbarzadeh, A., Neville, A., Morina, A., Wilson, M.C. Modelling tribochemistry in the mixed lubrication regime. Tribology International 132:265-274 (2019).

[15] Chen, Z., Gu, C., Tian, T. Modeling of Formation and Removal of ZDDP Tribofilm on Rough Surfaces. Tribology Letters 69:1-9 (2021).

[16] Hardy, W.B., Doubleday, I. Boundary lubrication.- The paraffin series. Proceedings of the Royal Society of London Series A, Containing Papers of a Mathematical and Physical Character 100:550-574 (1922).

[17] Buyanovskii, I. Boundary lubrication by an adsorption layer. Journal of friction and wear 31:33-47 (2010).

[18] Greenwood, J., Tripp, J. The contact of two nominally flat rough surfaces. Proceedings of the institution of mechanical engineers 185:625-633 (1970).

[19] (!!! INVALID CITATION !!! ).

[20] Patir, N., Cheng, H. An average flow model for determining effects of three-dimensional roughness on partial hydrodynamic lubrication. Journal of lubrication Technology 100:12-17 (1978).

[21] Roelands, C.J.A., Winer, W.O., Wright, W.A. Correlational Aspects of the Viscosity-TemperaturePressure Relationship of Lubricating Oils(Dr In dissertation at Technical University of Delft, 1966). Journal of Lubrication Technology 93:209-210 (1971).

[22] Bowden, F.P., Leben, L. The friction of lubricated metals. Philosophical Transactions of the Royal Society of London Series A, Mathematical and Physical Sciences 239:1-27 (1940).

[23] Bowden, F.P., Bowden, F.P., Tabor, D.: The friction and lubrication of solids. Oxford university press, (2001)

[24] Roshan, R., Priest, M., Neville, A., Morina, A., Xia, X., Warrens, C.P., et al. Friction modelling in boundary lubrication considering the effect of MoDTC and ZDDP in engine oils. Tribology Online 6:301-310 (2011).

[25] Patir, N., Cheng, H. Application of average flow model to lubrication between rough sliding surfaces. (1979)

[26] Pasaribu, H., Lugt, P.M. The composition of reaction layers on rolling bearings lubricated with gear oils and its correlation with rolling bearing performance. Tribology transactions 55:351-356 (2012).

[27] Fujita, H., Glovnea, R., Spikes, H. Study of zinc dialkydithiophosphate antiwear film formation and removal processes, part I: experimental. Tribology transactions 48:558-566 (2005).

[28] Bayat, R., Lehtovaara, A. Tribofilm Formation of Simulated Gear Contact Along the Line of Action. Tribology Letters 69:1-11 (2021).

[29] Bec, S., Tonck, A., Georges, J.M., Coy, R.C., Bell, J.C., Roper, G.W. Relationship between mechanical properties and structures of zinc dithiophosphate anti-wear films. Proceedings of the Royal Society A: Mathematical, Physical and Engineering Sciences 455:4181-4203 (1999).

[30] Nehme, G., Mourhatch, R., Aswath, P.B. Effect of contact load and lubricant volume on the properties of tribofilms formed under boundary lubrication in a fully formulated oil under extreme load 
conditions. Wear 268:1129-1147 (2010).

[31] Aktary, M., McDermott, M.T., McAlpine, G.A. Morphology and nanomechanical properties of ZDDP antiwear films as a function of tribological contact time. Tribology Letters 12:155-162 (2002).

[32] Taylor, R.I. Tribology and energy efficiency: from molecules to lubricated contacts to complete machines. Faraday discussions 156:361-382 (2012).

[33] Demmou, K., Bec, S., Loubet, J.-L., Martin, J.-M. Temperature effects on mechanical properties of zinc dithiophosphate tribofilms. Tribology international 39:1558-1563 (2006).

[34] Pereira, G., Munoz-Paniagua, D., Lachenwitzer, A., Kasrai, M., Norton, P.R., Capehart, T.W., et al. A variable temperature mechanical analysis of ZDDP-derived antiwear films formed on 52100 steel. Wear 262:461-470 (2007).

[35] Tse, J.S., Song, Y., Liu, Z. Effects of Temperature and Pressure on ZDDP. Tribology Letters 28:4549 (2007).

[36] Bosman, R., Schipper, D.J. Mild wear prediction of boundary-lubricated contacts. Tribology letters 42:169-178 (2011).

[37] Demmou, K., Bec, S., Loubet, J.-L. Effect of hydrostatic pressure on elastic properties of ZDTP tribofilms. arXiv preprint arXiv:07064235 (2007).

[38] Salinas Ruiz, V.R., Kuwahara, T., Galipaud, J., Masenelli-Varlot, K., Hassine, M.B., Héau, C., et al. Interplay of mechanics and chemistry governs wear of diamond-like carbon coatings interacting with ZDDP-additivated lubricants. Nature Communications 12:1-15 (2021).

[39] Fujita, H., Spikes, H. The formation of zinc dithiophosphate antiwear films. Proceedings of the Institution of Mechanical Engineers, Part J: Journal of Engineering Tribology 218:265-278 (2004).

[40] Bosman, R., de Rooij, M.B. Transient thermal effects and heat partition in sliding contacts. (2010).

[41] Ueda, M., Kadiric, A., Spikes, H. Influence of Steel Surface Composition on ZDDP Tribofilm Growth Using Ion Implantation. Tribology Letters 69:1-14 (2021).

[42] Dichu, Chun, Wang, Cayetano, Espejo, Jiugen, et al. Understanding the friction reduction mechanism based on the molybdenum disulfide tribofilm formation and removal. Langmuir : the ACS journal of surfaces and colloids (2018).

[43] Rydel, J.J., Pagkalis, K., Kadiric, A., Rivera-Díaz-del-Castillo, P.E.J. The correlation between ZDDP tribofilm morphology and the microstructure of steel. Tribology International:S0301679X16304078 (2016).

[44] Xu, D., Wang, C., Espejo, C., Wang, J., Neville, A., Morina, A. Understanding the friction reduction mechanism based on molybdenum disulfide tribofilm formation and removal. Langmuir 34:13523-13533 (2018).

[45] Cyriac, F., Yi, T.X., Poornachary, S.K., Chow, P.S. Behavior and interaction of boundary lubricating additives on steel and DLC-coated steel surfaces. Tribology International 164:107199 (2021).

[46] Dawczyk, J., Morgan, N., Russo, J., Spikes, H. Film thickness and friction of ZDDP tribofilms. Tribology Letters 67:1-15 (2019).

[47] Azam, A., Dorgham, A., Parsaeian, P., Morina, A., Neville, A., Wilson, M.C. The mutual interaction between tribochemistry and lubrication: Interfacial mechanics of tribofilm. Tribology International 135:161-169 (2019).

[48] Zhao, Y., Geng, Z., Li, D., Wang, L., Lu, Z., Zhang, G. An investigation on the tribological properties of graphene and ZDDP as additives in PAO4 oil. Diamond and Related Materials 120:108635 (2021).

[49] Spikes, H. Stress-augmented thermal activation: Tribology feels the force. Friction 6:1-31 (2018). 